\title{
Molecular subtyping improves diagnostic stratification of patients with primary breast cancer into prognostically defined risk groups
}

\author{
Katharine Yao ${ }^{1} \cdot$ Robert Goldschmidt $^{1} \cdot$ Mary Turk $^{1} \cdot$ Jelle Wesseling $^{3}$ •

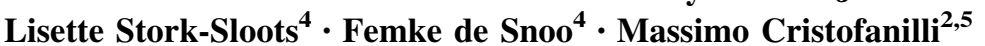

Received: 25 August 2015/Accepted: 24 September 2015/Published online: 30 September 2015

(C) The Author(s) 2015. This article is published with open access at Springerlink.com

\begin{abstract}
Combined use of MammaPrint and a molecular subtyping profile (BluePrint) identifies disease subgroups with marked differences in long-term outcome and response to neo-adjuvant therapy. The aim of this study was to evaluate the prognostic value of molecular subtyping using MammaPrint and BluePrint in women with earlystage breast cancer (BC) treated at US institutions following National Comprehensive Cancer Network standard guidelines. Tumor samples were collected from stage 1-2B consecutively diagnosed BC patients $(n=373)$ who underwent lumpectomy or mastectomy with an axillary staging procedure between 1992 and 2010 at two institutes (NorthShore University HealthSystem and Fox Chase Cancer Center) in the United States of America, with a median follow-up time of 9.5 years. MammaPrint low-risk patients had a 10-year DMFS of $96 \%$ (95 \%CI 92.8-99.4), while MammaPrint high-risk patients had a 10-year DMFS of $87 \%$ (95\%CI 81.9-92.1) with a hazard ratio of 3.62 (95\%CI 1.38-9.50) $(p=0.005)$. Uni- and multivariate analyses included age, tumor size, grade, ER, and Her2; in
\end{abstract}

Katharine Yao

kyao@northshore.org

Femke de Snoo

fdesnoo@medex15.com

1 NorthShore University HealthSystem, 2650 Ridge Ave. Suite 1155, Evanston, IL 60201, USA

2 Fox Chase Cancer Center, Philadelphia, PA, USA

3 Netherlands Cancer Institute, Amsterdam, The Netherlands

4 Agendia NV, Amsterdam, The Netherlands

5 Robert Lurie Cancer Center, Northwestern University, Chicago, IL, USA multivariate analysis, MammaPrint reached near-significance (HR $3.01 ; p$ 0.08). When comparing BluePrint molecular subtyping with clinical stratification, the prognosis (10-year DMFS) was significantly different in 10-year DMFS between the different molecular subtypes $(p<0.001)$. This retrospective study with 10 -year followup data provides valuable insight into prognosis of patients with primary $\mathrm{BC}$ comparing clinical with molecular subtyping. The BluePrint molecular stratification assay identifies patients with significantly different outcomes compared with standard clinical molecular stratification.

Keywords Breast cancer - Molecular subtyping . Molecular assay $\cdot$ Prognosis

\section{Introduction}

Improved understanding of the molecular phenotypes of breast cancer (BC) has already shown prognostic and predictive value and, when prospectively applied, could have dramatic implications in establishing a more personalized approach to the management of early-stage breast cancer. The St. Gallen Expert Consensus Guidelines for the recommendations of treatment in early-stage BC have recognized the importance of disease subtype classification and have included the surrogate pathology-based subtype classification definitions in lieu of medically accepted molecular classification techniques [1].

The rational-based method (supervised training) for development of the 80-gene molecular subtyping profile (BluePrint) is inherently different from the groundbreaking research used originally to unveil the existence of molecular subgroups using gene expression unsupervised clustering [2]. 
The 80-gene molecular subtyping profile (BluePrint) has been developed to provide a molecular classification technique based on gene expression of three functional and targetable pathways that distinguish Luminal-type versus HER-2-type, versus Basal-type. A rational-based method was used to ensure a robust and reproducible profile with concordant immunohistochemistry (IHC)/fluorescent in situ hybridization (FISH)-assessed and mRNA-assessed training samples for the estrogen receptor (ER), progesterone receptor (PR), and HER2. BluePrint ${ }^{\circledR}$ determines the mRNA expression levels of 80 genes that best discriminate between luminal-type, HER2-type, and basal-type tumors and was validated using four independent validation cohorts consisting of 784 patients [3]. The luminal subtype can be further divided into luminal-type A (low risk) and luminal-type B (high risk) using MammaPrint ${ }^{\circledR}$.

The clinical utility of BluePrint molecular classification was demonstrated in a previously published retrospective analysis of four pooled neo-adjuvant studies. In that cohort, 90 of $437(21 \%)$ patients were classified as luminal-type A. These patients, who could not be identified by standard IHC/FISH for ER, PR, HER2 biomarkers, demonstrated a distant metastasis-free survival rate of $93 \%$ at 5 years and showed little, if any, benefit from chemotherapy (the pCR rate was only $6 \%$ in this group) [4].

The aim of the current retrospective study was to evaluate the prognostic implication of accurate molecular subtyping using MammaPrint and BluePrint in women with early-stage BC treated in the adjuvant setting with 10-year follow-up at two US Institutions following National Comprehensive Cancer Network (NCCN) standard guidelines.

\section{Patients and methods}

\section{Patients and tumor samples}

Frozen $(n=205)$ and formalin-fixed paraffin-embedded (FFPE) tissue $(n=168)$ tumor samples were collected from stage 1-2B consecutively diagnosed $\mathrm{BC}$ patients who underwent lumpectomy or mastectomy with an axillary staging procedure between 1992 and 2010 at two institutes [NorthShore University HealthSystem $(n=275)$ and Fox Chase Cancer Center $(n=98)]$ in the United States of America. The median follow-up time of this patient cohort was 9.5 years. Clinical and histopathological data from all patients were collected.

\section{Local pathological assessment of ER, PR, and Her2}

Methods and cutoffs for local assessment of ER, PR, and HER2 varied during the years encompassed by this study.
At NorthShore, a $20 \%$ staining threshold was used to determine ER and PR status until 2002, at which time a $1 \%$ threshold was instituted. Different antibody clones were also utilized over this time period, including Dako 6F11, 1D5, and SP1 for ER and 1294 for PR. For HER2 testing, older cases were analyzed using the Dako Herceptest. Subsequently, IHC using the Zymed Tab 250 or Ventana 4B5 antibody followed by FISH testing for equivocal cases was performed. IHC was scored as 0-3+ using standard criteria. A single probe FISH test (Ventana) was scored as either negative $(<4$ signals/cell), equivocal (4-6 signals/cell), or positive ( $>6$ signals/cell). The most recent cases were analyzed using the INFORM (Ventana) chromogenic in situ dual probe system and scored based on the HER2:CEP17 ratio as negative (ratio $<1.8$ ), equivocal (ratio 1.8-2.2), or positive (ratio $>2.2$ ).

\section{Molecular subtyping}

Microarray analysis for obtaining the BluePrint and MammaPrint signatures was done by staff at Agendia Laboratories, Amsterdam, who were blinded to clinical and pathological data. Expression data were quantified using Feature Extraction software [5]. Four distinct molecular subgroups-luminal A-type, luminal B-type, basal-type, and HER2-type-were identified and used for further analysis. In this study, we defined luminal A-type tumors as luminal-type by BluePrint with a low-risk score by MammaPrint and luminal B-type tumors as BluePrint luminal-type with a MammaPrint high-risk score.

\section{Central re-assessment of IHC/FISH}

Patients who had a discordant subtype classification [BluePrint vs. clinical (IHC/FISH)] were centrally reassessed for ER, PR, and Her2.

In the central laboratory, ER and PR statuses were assessed on FFPE tissue by IHC using the ER/PR PharmDX kit (Dako, Glostrup, Denmark). Tumors were classified as ER- or PR-positive when $\geq 1 \%$ invasive tumor cells showed definite nuclear staining, irrespective of staining intensity [6]. HER2 expression was evaluated with the HercepTest kit (Dako) and scored as $0,1+, 2+$, or 3+, according to the FDA scoring system. Tumors scored as $2+$ were re-tested with SISH using the PathVysion HER2 DNA probe kit (Vysis-Abbott, Chicago, USA). Cases were considered HER2-positive if scored $3+$ by IHC and/or amplified by SISH (ratio $>2$ ).

\section{Statistical analyses}

The primary end point was distant metastases-free survival (DMFS), which was defined as the probability that patients 
remain free of distant metastases as the first event; data on all other patients were censored on the date of the last follow-up visit or date of death. Data were analyzed from the date of surgery to the time of the first event or the date on which data were censored, according to the KaplanMeier method, and the curves were compared with use of the log-rank test. In order to determine the independence of MammaPrint to clinic-pathological variables in predicting an individual's risk of developing distant metastases, we analyzed the results using univariate and multivariate analysis. Variables included MammaPrint; age; tumor size; grade; ER status; and HER2 status. All calculations were performed with SPSS statistical package version 22.0 (SPSS, Chicago, IL).

\section{Results}

The clinical characteristics of the 373 patients (median age 56 range 28-97 years) are shown in Table 1. Two-hundred and seventy-five (74\%) patients had ER-positive disease, $62(17 \%)$ had Her2-positive, and $73(20 \%)$ triple-negative disease. Two-hundred and sixty-eight (72\%) patients had lymph node negative disease. A total of $217(58 \%)$ patients received adjuvant chemotherapy (CT) and 244 (65\%) adjuvant endocrine therapy (ET). Since most patients were diagnosed prior to 2005 , only 11 out of 62 (18\%) patients with Her2-positive disease received adjuvant trastuzumab $(\mathrm{H})$. For $64 \%$, the chemotherapy regimen was known and consisted of standard anthracyclineregimens. Most patients $(84 \%)$ received AC (doxorubicin/cyclophosphamide)/FAC (cyclophosphamide/doxorubicin/fluorouracil) or FEC (cyclophosphamide/ epirubicin/fluorouracil), followed by TAC (docetaxel/ doxorubicin/cyclophosphamide) $(12 \%)$, and $\mathrm{CMF}$ (cyclophosphamide/methotrexate/fluorouracil) (6\%).

MammaPrint classified $152(41 \%)$ tumors as low-risk and $221(59 \%)$ as high-risk. The combined analysis of BluePrint and MammaPrint molecular subtyping classified $150(40 \%)$ tumors as luminal A-type, 102 (27\%) patients as luminal B-type, 37 (10 \%) tumors as HER2-type, and 84 (23\%) tumors as basal-type. Surrogate pathology-based molecular classification using IHC/FISH for ER, PR, and Her2 identified 238 (64\%) tumors as HR-positive ("luminal"), 62 (17\%) tumors as Her2-positive, and 73 (20\%) tumors as triple-negative ("basal").

Interestingly, 17 out of 238 (7\%) IHC/FISH HR-positive ("surrogate luminal") tumors were identified not classified as luminal-type by BluePrint and were re-classified, two were HER2-type and 15 basal-type. Thirty-three out of $62(53 \%)$ identified by IHC/FISH as Her2+ tumors
Table 1 Clinical characteristics

\begin{tabular}{|c|c|}
\hline Characteristic & $\begin{array}{l}\text { Number of patients } \\
\text { (percentage) }\end{array}$ \\
\hline Age & 56 years (range $28-97$ ) \\
\hline \multicolumn{2}{|l|}{ Ethnicity } \\
\hline Caucasian & $320(86 \%)$ \\
\hline African American & $23(6 \%)$ \\
\hline Asian & $9(2 \%)$ \\
\hline Other & $21(6 \%)$ \\
\hline \multicolumn{2}{|l|}{ Stage } \\
\hline Stage 1 & $167(45 \%)$ \\
\hline Stage $2 \mathrm{~A}$ & $135(36 \%)$ \\
\hline Stage $2 B$ & $71(19 \%)$ \\
\hline \multicolumn{2}{|l|}{ Lymph nodes } \\
\hline 0 & $268(72 \%)$ \\
\hline $1-3$ & $84(23 \%)$ \\
\hline$>3$ & $17(5 \%)$ \\
\hline Unknown & $4(1 \%)$ \\
\hline \multicolumn{2}{|l|}{ Grade } \\
\hline Grade 1 & $67(18 \%)$ \\
\hline Grade 2 & $121(32 \%)$ \\
\hline Grade 3 & $180(48 \%)$ \\
\hline Grade unknown & $5(1 \%)$ \\
\hline \multicolumn{2}{|l|}{ Surgery } \\
\hline Mastectomy & $106(28 \%)$ \\
\hline Lumpectomy & $265(71 \%)$ \\
\hline Surgery unknown & $2(1 \%)$ \\
\hline \multicolumn{2}{|l|}{ Local IHC/FISH } \\
\hline ER-positive & $275(74 \%)$ \\
\hline PR-positive & $208(56 \%)$ \\
\hline Her2-positive & $62(17 \%)$ \\
\hline \multicolumn{2}{|l|}{ Treatment } \\
\hline No treatment & $35(9 \%)$ \\
\hline ET & $121(32 \%)$ \\
\hline $\mathrm{CT}$ & $89(24 \%)$ \\
\hline $\mathrm{ET}+\mathrm{CT}$ & $117(31 \%)$ \\
\hline $\mathrm{CT}+\mathrm{H} \pm \mathrm{ET}$ & $11(3 \%)$ \\
\hline \multicolumn{2}{|l|}{$\mathrm{CT}$ regimen } \\
\hline $\mathrm{AC} / \mathrm{FAC} / \mathrm{FEC}$ & $115(53 \%)$ \\
\hline TAC & $16(7 \%)$ \\
\hline $\mathrm{CMF}$ & $8(4 \%)$ \\
\hline Unknown* & $78(36 \%)$ \\
\hline
\end{tabular}

* No details about the CT regimen were known for Fox Chase Cancer Center patients

IHC immuno histochemistry, FISH fluorescent in situ hybridisation, $E R$ estrogen receptor, $P R$ progesterone receptor, HER2 human epidermal growth factor receptor 2, ET endocrine treatment, $C T$ chemotherapy treatment, $H$ trastuzumab, $A$ doxorubicin, $C$ cyclophosphamide, $F$ fluorouracil, $E$ epirubicin, $T$ docetaxel or paclitaxel, $M$ methotrexate 
were re-classified by BluePrint as luminal-type (24) and basal-type (9). Moreover, 13 out of 73 (18\%) triple-negative ("surrogate basal") tumors were identified by BluePrint as luminal-type (7) and HER2-type (6) (Fig. 1).

\section{MammaPrint 10-year DMFS validation}

The Kaplan-Meier survival curves (Fig. 2a) show that MammaPrint low-risk patients had a 10-year DMFS of $96 \%$ (95 \%CI 92.8-99.4), while MammaPrint high-risk patients had a 10-year DMFS of $87 \%(95 \%$ CI 81.9-92.1) with a HR of 3.62 (95 \%CI 1.38-9.50) ( $p=0.005)$. In detail, $38 \%$ of MammaPrint low-risk patients received adjuvant $\mathrm{CT}$ and $91 \%$ received ET. Furthermore, $72 \%$ of MammaPrint high-risk patients received adjuvant $\mathrm{CT}$ and $48 \%$ received ET. MammaPrint low-risk patients $(n=93)$ who did not receive adjuvant CT had a 10-year DMFS of $98 \%(95 \% \mathrm{CI}$ $94.0-100)$ and MammaPrint high-risk patients $(n=60)$ who did not receive adjuvant CT had a 10-year DMFS of $85 \%$ (95 \% CI 74.8-95.6), data not shown.

Uni-and multivariate analyses were done with the following clinical variables: age, tumor size, grade, ER, and Her2. Of the clinical variables, grade (3 vs. $1 \& 2$ ) (HR 3.14; $p$ 0.006), and ER (HR 0.44; $p 0.03$ ) reached significance for 10-year DMFS. Age, tumor size, and Her2 were not significantly correlated with time to Distant Metastases. Grade and ER lost their significance in the multivariate analysis, and only MammaPrint reached near-significance (HR 3.01; $p$ 0.08) (Table 2).

A sub-analysis of MammaPrint in patients with $\mathrm{IHC} /$ FISH HR-positive and Her2-negative disease also significantly predicted 10-year DMFS with a HR of 2.91 (95 \% CI $0.97-8.68 ; p=0.045)$. MammaPrint low-risk patients
Fig. 1 Pie charts depicting restratification of patients first classified according to Clinical (IHC/FISH) subtyping and then re-classified by molecular subtyping with MammaPrint/ BluePrint

\section{Clinical (IHC/FISH) total $n=373$

"Luminal"
ER+ AND/OR PgR+
AND HER2-
$\mathrm{n}=238$

HER2+/HR+ ER+ AND/OR PgR+ AND HER2+ $\mathrm{n}=38$

\section{HER2+/HR- ER+ AND PgR- AND HER2+ $\mathrm{n}=24$}

Triple Negative PgR-AND ER-AND HER2$n=73$

\section{MammaPrint/BluePrint}
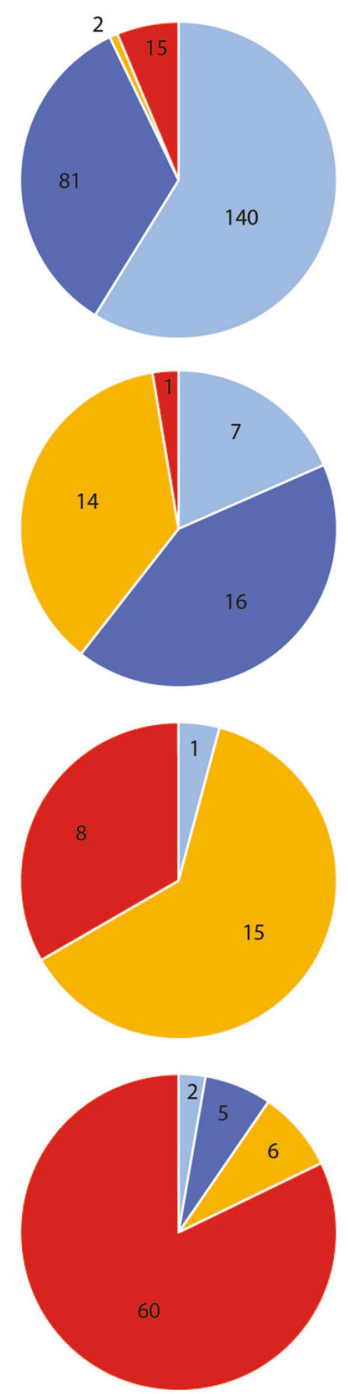

Luminal A-type

- Luminal B-type

- HER2-type

- Basal-type 
$(n=142)$ had a 10-year DMFS of $96 \%(95 \%$ CI 92.4-99.4), while MammaPrint high-risk patients $(n=96)$ had a 10-year DMFS of $87 \%$ (95\%CI 78.9-95.3)
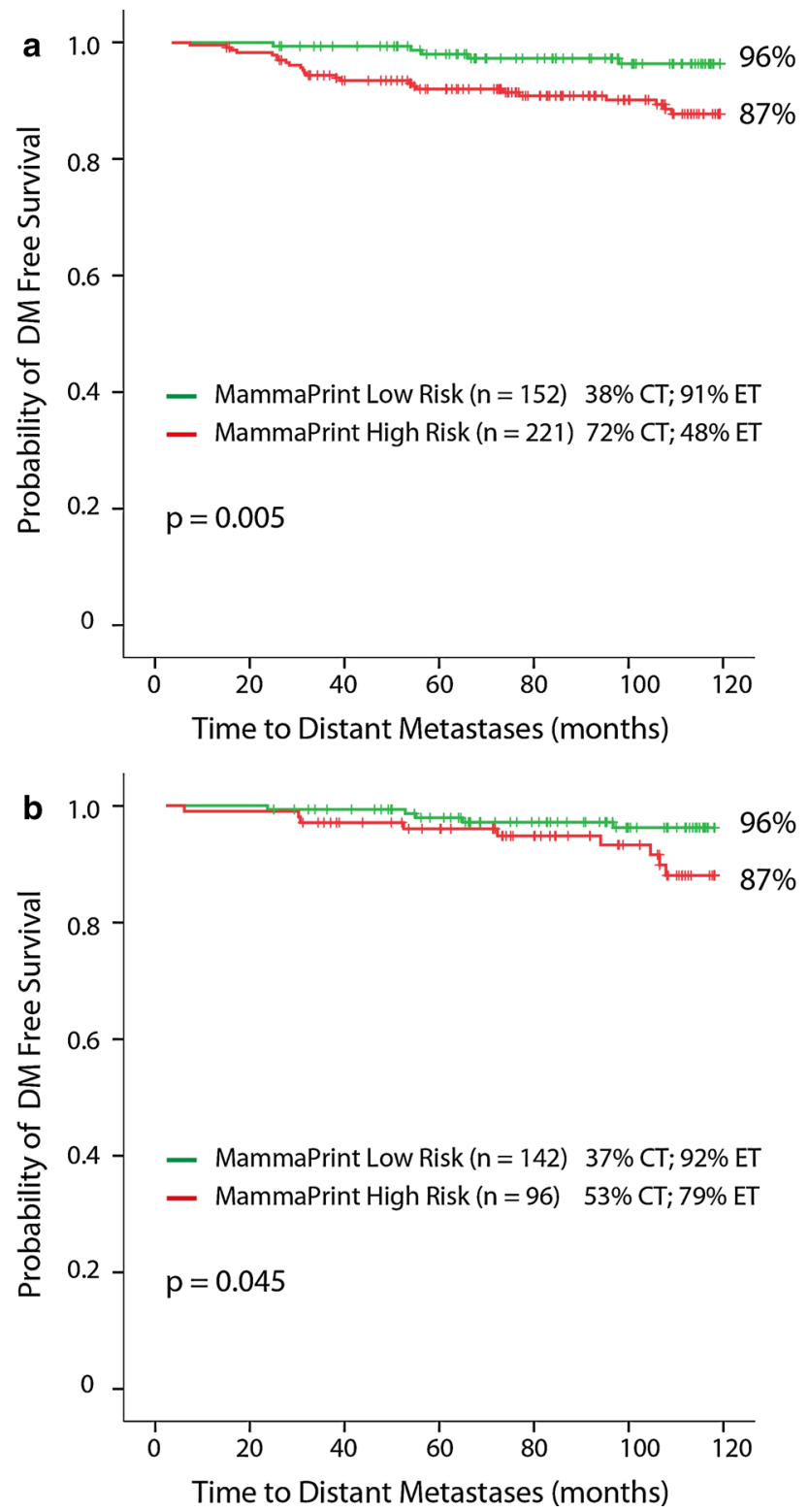

Fig. 2 a MammaPrint 10-year DMFS all patients. b MammaPrint 10-year DMFS IHC/FISH HR+ HER2- patients
(Fig. 2b). Moreover, $37 \%$ of these MammaPrint low-risk patients received adjuvant $\mathrm{CT}$; $92 \%$ received ET; and $53 \%$ of MammaPrint high-risk patients received adjuvant CT, while $79 \%$ received ET.

\section{Molecular versus clinical subtype 10-year DMFS}

There is a significant difference in 10-year DMFS among different molecular subtypes $(p<0.001)$ (Fig. 3a). Luminal A-type has a 10-year DMFS of $96 \%$ (95\%CI 92.8-99.4) compared to $91 \%(95 \% \mathrm{CI} 84.0-98.2)$ in luminal B-type patients; $75 \%$ (95\%CI 60.5-89.1) in HER2-type patients, and $88 \%$ (95 \%CI 79.5-95.5) in basal-type patients. A sub-analysis of luminal A-type patients who did not receive adjuvant CT $(n=92)$ shows a 10-year DMFS of $97 \%$ (95\%CI 93.9-100) (two events at 65 months), while luminal B-type patients who did not receive adjuvant CT $(n=44)$ had a 10 -year DMFS of $88 \%$ (95 \%CI 75.9-99.1). Thirty-eight percent of luminal A patients received adjuvant CT. We had the opportunity to evaluate the prognostic value of HER-2 molecular subtype in relation to trastuzumab adjuvant treatment. Approximately, $11 \%$ of HER2-type patients received adjuvant trastuzumab and they had a 10-year DMFS of $100 \%$. On the contrary, HER2-type patients who did not receive adjuvant trastuzumab $(n=33)$ had a 10-year DMFS of $72 \%$ (95\%CI 56.5-87.5), data not shown.

Figure $3 \mathrm{~b}$ shows the analysis of the same patients now stratified according to surrogate pathology-based clinical subtyping. The KM-curve shows no significant difference between the IHC/FISH subgroups. Patients with HR-positive ("surrogate luminal") BC had a 10-year DMFS of $93 \%$ (95\%CI 88.9-96.3) compared to $86 \%$ (95\%CI 76.8-95.2) in patients with Her2-positive breast cancer, and $90 \%$ (95 \% CI 82.8-97.0) in patients with triple-negative ("surrogate basal") breast cancer. Again, $18 \%$ of Her2-positive patients received adjuvant trastuzumab. Her2-positive patients who received adjuvant trastuzumab $(n=11)$ had a 10 -year DMFS of $100 \%$, while Her2positive patients who did not receive adjuvant trastuzumab $(n=51)$ had a 10-year DMFS of $83 \% \quad(95 \% \mathrm{CI}$ 72.6-93.8), data not shown.

Table 2 Univariate and multivariate analysis of risk of 10-year DMFS

\begin{tabular}{lllllll}
\hline Variable & $p$ value & Univariate HR & Univariate 95 \% CI & $p$ value & Multivariate HR & Multivariate 95 \% CI \\
\hline MammaPrint, (High vs. low risk) & 0.009 & 3.623 & $1.382-9.499$ & 0.080 & 3.011 & $0.877-10.333$ \\
Age, continuous & 0.954 & 1.001 & $0.974-1.028$ & 0.440 & 1.011 & $0.984-1.039$ \\
Tumor size & 0.828 & 0.994 & $0.944-1.047$ & 0.780 & 0.991 & $0.932-1.054$ \\
Grade (3 vs. 1\&2) & 0.006 & 3.139 & $1.388-7.097$ & 0.411 & 1.553 & $0.544-4.439$ \\
ER (pos vs. neg) & 0.030 & 0.440 & $0.210-0.923$ & 0.559 & 0.773 & $0.326-1.834$ \\
HER2 (pos vs. neg) & 0.087 & 2.036 & $0.901-4.597$ & 0.412 & 1.428 & $0.609-3.347$ \\
\hline
\end{tabular}





Fig. 3 a 10-year DMFS MammaPrint/BluePrint molecular subgroups. b 10-year DMFS IHC/FISH subgroups

\section{Central re-assessment of discordant BluePrint and clinical (IHC/FISH) subtypes}

Sixty-three out of $373(17 \%)$ tumors had a discordant molecular subtype compared to the surrogate pathologybased IHC/FISH subtype. Fifty-three out of 63 re-classified tumors were available for central re-assessment of ER, PR, and HER 2 by IHC/SISH. The results are shown in Table 3. There were 15 tumors locally classified as HR+/HER2("surrogate luminal") and not luminal-type by BluePrint. Central re-assessment confirmed the local result for eight cases and the BluePrint result for seven cases. There were 27 tumors locally classified as Her2+ and not HER2-type by BluePrint. Central re-assessment confirmed the local result for seven cases and the BluePrint result for 15 cases. There were 11 tumors locally classified as triple-negative ("surrogate basal") and not basal-type by BluePrint. Central re-assessment confirmed the local result for four cases and the BluePrint result for six cases. Of all centrally assessed Her2-positive tumors $(n=10)$, six were re-classified as luminal-type by BluePrint molecular classification. These six tumors were strongly ER-positive ( $\geq 80 \%)$, data not shown.

\section{Discussion}

This retrospective study demonstrated that MammaPrint is able to accurately differentiate between patients at a low and a high risk of distant metastases up to 10 years after diagnosis. Patients with a MammaPrint low-risk result have an excellent 10-year DMFS survival. Remarkably, the results were consistent among both group of patients, the cohort that did not receive adjuvant treatment and the one for which treatment was administered suggesting prognostic and predictive value in the studied population. These results provide additional confirmation of the clinical utility for the MammaPrint test as a means of correctly identifying those patients who may not benefit from the treatment with adjuvant chemotherapy. The use of genetic signatures such as MammaPrint for determining which patients can forego adjuvant chemotherapy treatment has been accepted by most early-stage $\mathrm{BC}$ treatment guidelines $[1,7]$. However, the most optimal diagnostic technique for stratification of patients for determining endocrine, HER2targeted treatment, and correct treatment allocation for "basal-type" patients is openly being questioned in such guidelines.

The current study on comparison of surrogate versus gene-expression-based molecular subtype classification of tumors with 10-year outcome has been performed to aid in the quest for identifying optimal molecular stratification assays. The study shows that molecular subtyping with BluePrint and MammaPrint based on a gene expression assay leads to a more accurate classification of tumors with clinically significant prognostic stratification compared to surrogate pathology-based clinical subtypes. Luminal A patients have an excellent 10 years DMFS of $96 \%$ DMFS, and Luminal B patients have a 10-year DMFS of $91 \%$ and include also patients who would otherwise be treated as Her2 or triple-negative patients. In total, 63 out of $373(17 \%)$ patients had a different molecular subtype compared to the surrogate-based pathologically determined subtype. This percentage of discordance is in line with percentages seen in other studies $[4,8]$. The discrepancy between molecular- and 
Table 3 Central re-assessment of discordant BluePrint and clinical (IHC/FISH) subtype cases

\begin{tabular}{llllll}
\hline Local pathology (IHC/FISH) & BluePrint & \multicolumn{2}{l}{ Central pathology (IHC/FISH) } & \\
\cline { 3 - 6 } & & HR+/Her2- & Her2 + & TN & Total \\
\hline HR+/Her2- & HER2-type & 1 & 1 & 0 & 2 \\
HR+/Her2- & Basal-type & 7 & 0 & 6 & 13 \\
Her2+ & Luminal-type & $12^{*}$ & 6 & 1 & 19 \\
Her2+ & Basal-type & 4 & 1 & 3 & 8 \\
TN & Luminal-type & 4 & 0 & 2 & 6 \\
TN & HER2-type & 1 & $2 *$ & 2 & 5 \\
\hline
\end{tabular}

* Diagnosed between 1996 and 2009 (11 out of 14 diagnosed before 2003) surrogate pathology-based clinical subtyping is reasonable because the two methods are based on different genotypic features of the tumor; where hormone receptors and HER2 are measured individually, BluePrint is designed according to a rational-based method such that it captures the functional underlying biologic pathway regulated by ER, PR, and HER-2 [3].

Six percent of patients with surrogate pathology-based "luminal" (HR+/Her2-) disease were classified as basaltype BluePrint. This percentage is slightly higher than the $2 \%$ reported earlier in the MINDACT study, in which tumors were centrally assessed for the pathology-based subtypes and compared to expression-based BluePrint subtypes. The higher percentage in the current study may be explained by the higher threshold for ER-positivity used prior to 2002 in one of the 2 institutions.

The occurrence of IHC ER+ patients classified as basaltype by BluePrint was previously explained by a relatively high expression of the dominant-negative ER $\alpha$-splice variant ERD7 in ER-positive/basal-type tumors as compared to ER-positive/luminal-type tumors $(p<0.0001)$ [9]. Expression of the dominant-negative ER $\alpha$ variant ERD7 provides a rationale as to why tumors are identified as BluePrint basal-type, while staining ER-positive by IHC; the BluePrint test appears to measure ER activity independent of the ER $\alpha$ mRNA expression level itself. These tumors may lack a functional response to estrogen and consequently may not respond to endocrine manipulation with profound implications for management of these patients. Similarly, Iwamoto et al. found that most of the 1-9 \% IHC ER-positive tumors show molecular features similar to ER-negative basal-like tumors [10], who suggest to treat with the safest clinical approach using both adjuvant endocrine therapy and chemotherapy in this rare subset of patients. Another study also found that low-ERstaining tumors were clinic-pathologically more similar to ER-negative than to ER-positive tumors; $88 \%$ of lowstaining tumors were basal-like or HER2 enriched. Only those tumors expressing $10 \%$ ER-positive cells were classified as luminal A subtype [11].
Another group of patients with discordant disease between surrogate pathology-based and molecular-based subtyping is the so-called triple-positive disease patient group: hormonal-positive and Her $2+$ disease. BluePrint molecular subtyping re-classifies around $50 \%$ of these patients as luminal-type, with improved prognostic utility: these patients have an outcome in line with the expected outcome of Luminal patients. The BluePrint HER2-type patients, however, have worse outcome compared to clinical Her2-positive patients, and this result was particularly striking in patients who did not receive trastuzumab. These data can therefore be regarded as a validation of BluePrint molecular subtyping as a means of identifying specific patient groups for treatment allocation. The negative results for the identification of patients who would have no benefit from trastuzumab by PAM50-based molecular subgroups in the NSABP B31 trial presented at SABCS may have closed the door for molecular subtyping [12]. The development of the two molecular subtyping profiles is inherently different, where PAM50 was based on unsupervised clustering versus BluePrint which was developed on a rational-based method, leading to a functional subtyping profile [3]. PAM50 classifies approximately $10 \%$ of clinical luminal/Her2-negative patients as HER2 enriched, and $17 \%$ of triple-negative patients as Her2 enriched, potentially diluting the sensitivity of identifying anti-Her2 treatment sensitivity. BluePrint molecular subtyping classifies less than $1 \%$ of clinical luminal/HER2-negative as HER2-type and $1 \%$ of triple-negative patients, allowing the predictive sensitivity for anti Her2 treatment to be significant between molecularly versus clinically identified subgroups [8]. In a direct comparative study where patients are classified according to BluePrint as well as PAM50, the concordance was only $59 \%$, indicating that these assays assign tumors differently [13].

In conclusion, this retrospective non-randomized study provides valuable insight into the prognostic and predictive value of molecular testing in patients with early breast cancer. The unique aspect of the current study is the combination of both molecular tests allowing to explore the 
potential clinical applications. The increasing availability of targeted therapies for patients with both luminal and HER2 subtypes, and the increased use of prolonged adjuvant endocrine therapy require a more rational approach to patients' selection in order to maximize benefits, improve quality of life, and reduce costs.

Acknowledgments Whole genome analysis was provided in kind by Agendia.

\section{Compliance with ethical standards}

Conflict of Interest FdS and LS-S were employees of Agendia at the time of analysis. MC received honoraria from Agendia.

Open Access This article is distributed under the terms of the Creative Commons Attribution-NonCommercial 4.0 International License (http://creativecommons.org/licenses/by-nc/4.0/), which permits any noncommercial use, distribution, and reproduction in any medium, provided you give appropriate credit to the original author(s) and the source, provide a link to the Creative Commons license, and indicate if changes were made.

\section{References}

1. Goldhirsch A, Winer EP, Coates AS, Gelber RD, Piccart-Gebhart M, Thürlimann B, Senn HJ, Panel members (2013) Personalizing the treatment of women with early breast cancer: highlights of the St Gallen International Expert Consensus on the Primary Therapy of Early Breast Cancer 2013. Ann Oncol 24(9):2206-2223

2. Perou CM, Sørlie T, Eisen MB, van de Rijn M, Jeffrey SS, Rees CA, Pollack JR, Ross DT, Johnsen H, Akslen LA, Fluge O, Pergamenschikov A, Williams C, Zhu SX, Lønning PE, Børresen-Dale AL, Brown PO, Botstein D (2000) Molecular portraits of human breast tumours. Nature 406:747-752

3. Krijgsman O, Roepman P, Zwart W, Carroll JS, Tian S, de Snoo FA, Bender RA, Bernards R, Glas AM (2012) A diagnostic gene profile for molecular subtyping of breast cancer associated with treatment response. Breast Cancer Res Treat 133:37-47

4. Glück S, de Snoo F, Peeters J, Stork-Sloots L, Somlo G (2013) Molecular subtyping of early-stage breast cancer identifies a group of patients who do not benefit from neoadjuvant chemotherapy. Breast Cancer Res Treat 139(3):759-767

5. Delahaye LJM, Wehkamp D, Floore AN, Bernards R, van't Veer LJ, Glas AM (2013) Performance characteristics of the
MammaPrint ${ }^{\circledR}$ breast cancer diagnostic gene signature. Personal Med 10(8):801-811

6. Hammond ME, Hayes DF, Dowsett M, Allred DC, Hagerty KL, Badve S, Fitzgibbons PL, Francis G, Goldstein NS, Hayes M, Hicks DG, Lester S, Love R, Mangu PB, McShane L, Miller K, Osborne CK, Paik S, Perlmutter J, Rhodes A, Sasano H, Schwartz JN, Sweep FC, Taube S, Torlakovic EE, Valenstein P, Viale G, Visscher D, Wheeler T, Williams RB, Wittliff JL, Wolff AC (2010) American Society of Clinical Oncology/College of American Pathologists guideline recommendations for immunohistochemical testing of estrogen and progesterone receptors in breast cancer. J Clin Oncol 28(16):2784-2795

7. Senkus E, Kyriakides S, Penault-Llorca F, Poortmans P, Thompson A, Zackrisson S, Cardoso F (2013) ESMO Guidelines Working Group. Primary breast cancer: ESMO Clinical Practice Guidelines for diagnosis, treatment and follow-up. Ann Oncol 24(Suppl 6):vi7-vi23

8. Whitworth P, Stork-Sloots L, de Snoo FA, Richards P, Rotkis M, Beatty J, Mislowsky A, Pellicane JV, Nguyen B, Lee L, Nash C, Gittleman M, Akbari S, Beitsch PD (2014) Chemosensitivity predicted by BluePrint 80 -gene functional subtype and MammaPrint in the prospective Neoadjuvant Breast Registry Symphony Trial (NBRST). Ann Surg Oncol 21(10):3261-3267

9. Groenendijk FH, Zwart W, Floore A, Akbari S, Bernards R (2013) Estrogen receptor splice variants as a potential source of false-positive estrogen receptor status in breast cancer diagnostics. Breast Cancer Res Treat 140(3):475-484

10. Iwamoto T, Booser D, Valero V, Murray JL, Koenig K, Esteva FJ, Ueno NT, Zhang J, Shi W, Qi Y, Matsuoka J, Yang EJ, Hortobagyi GN, Hatzis C, Symmans WF, Pusztai L (2012) Estrogen receptor (ER) mRNA and ER-related gene expression in breast cancers that are $1 \%$ to $10 \%$ ER-positive by immunohistochemistry. J Clin Oncol 30(7):729-734

11. Deyarmin B, Kane JL, Valente AL, van Laar R, Gallagher C, Shriver CD, Ellsworth RE (2013) Effect of ASCO/CAP guidelines for determining ER status on molecular subtype. Ann Surg Oncol 20(1):87-93

12. Pogue-Geile KL, Song N, Jeong JH, Gavin PG, Kim SR, Blackmon NL, Finnigan M, Rastogi P, Fehrenbacher L, Mamounas EP, Swain SM, Wickerham DL, Geyer CE Jr, Costantino JP, Wolmark N, Paik S (2015) Intrinsic subtypes, PIK3CA mutation, and the degree of benefit from adjuvant trastuzumab in the NSABP trial B-31. J Clin Oncol 33(12):1340-1347

13. Bayraktar S, Royce M, Stork-Sloots L, de Snoo F, Glück S (2014) Molecular subtyping predicts pathologic tumor response in earlystage breast cancer treated with neoadjuvant docetaxel plus capecitabine with or without trastuzumab chemotherapy. Med Oncol 31(10):163 